\title{
Behaviour of Crocidura leucodon
}

\begin{abstract}
[The bicoloured white-tooted shrew. The behaviour and spatial, ethological and functional structure of a population]. N. A. Shchipanov and V. Yu. Oleinichenko. Nauka, Moscow, 1993, 135 pp, 43 figs, 13 tables. ISBN 5-02-005776-2. [In Russian]
\end{abstract}

This book is the first available monograph on the behaviour of a single shrew species, ie Crocidura leucodon (Hermann, 1780). It is divided into three parts. The first contains 8 chapters presenting eg the survey of behavioural patterns, reproductive and parental behaviours and differences in social and spatial behaviours of shrews kept in captivity in pairs or in groups. The second part concerns the etho-ecology of $C$. leucodon in the wild and consists of 6 chapters presenting eg the habitats inhabited by the species in Caucasus, space usage, spatial structure of populations, migrations and fluctuations in population density. In the two chapters of the third part, the authors discuss recent theories on mechanisms regulating population structures in mammals and they propose one such mechanism for populations of $C$. leucodon. In their theory, high population density inhibits aggressive and sexual behaviours (especially in males), which results in cessation of reproduction.

Although Soricidae have been intensively investigated for many years, very few monographs have been published. Perhaps the best known books on shrews are by Crowcroft (1957) - with a 'popular' style, based mainly on the author's own observations, and Churchfield (1990) - a recent compendium of the scientific knowledge on shrews. Therefore, the book by Shchipanov and Oleinichenko is worthy of readers' attention at least because of this rarity. However, the main value of the book for soricologists is its observations on rarely studied and almost unknown aspects of shrew biology. The authors have undertaken a very broad and penetrating analysis of behaviour of a single species. They accomplished this by way of large-scale field studies (CMR trapping, habitat analysis) and, simultaneously, various laboratory experiments with multiple trials in which ca 300 animals were used. Some experiments, on social interactions or mother-offspring bonds, lasted several months. (Of note are the very interesting enclosure system constructed by the authors and the methods of successfully keeping and breeding C. leucodon, both of which are described in detail). The authors were very attentive, patient and obviously were good at observation. They therefore obtained relatively broad knowledge of the species studied and many new and very interesting results. (Part of their observation have been published before in two papers - Shchipanov and Oleinichenko 1992a, b). This allowed them to present some generalizations concerning eg spatial and functional structures of a population.

The authors propose that an increase in population density inhibits breeding. They try to support this hypothesis (pages 66-67) by the results of laboratory experiments (animals kept in crowded enclosures failed to reproduce). However, this seems to be an artifact of cage keeping. Fortunately, the authors are prudent in this interpretation. They acknowledge that they do not know if such a mechanism realy functions in the wild. In my opinion, the authors place too much emphasis on the relationship between the different population densities and changes in social behaviour, whereas they neglect the influence of reproductive and non-reproductive seasons. Their field studies were carried out mostly in August and September, ie when adult C. leucodon probably switched from reproductive into non-reproductive conditions. This could explain the change in shrews' behaviour (disappearance of territoriality) and change of population structure from dispersed into clumped.

Although a considerable part of the results presented are so called 'first-hand' material, there is a lack of any statistical analysis of these data. Many statements and conclusions are based only on the authors' convictions and intuition or even single observations. Though such exemplary observations are usually very interesting and informative they cannot be the only arguments for general statements. 
The book is written in a rather 'wordy' style. Moreover, some statements and information are repeated several times. The authors discuss their findings in the context of well selected literature but some most recent and important papers by western authors are not included. On the other hand, the book is a good source of papers on shrews written in Russian.

There are some other shortcomings: It seems that the authors do not distinguish between home range and territory. In some tables (eg Table 7 and 8 ) values have incorrect totals. Some references to figures and tables are also incorrect.

Nevertheless, this is a very interesting book and it was a pity that it reached us so late and is written in Russian which makes it largely unavailable to western mammalogists. But it is worthy of recommendation because it is exceptional among the theriological literature. A revised edition in English is most desirable.

\section{References}

Churchfield S. (ed) 1990. The natural history of shrews. Christopher Helm, London: 1-192.

Crowcroft P. (ed) 1957. The life of the shrew. Max Reinhardt, London: 1-166.

Shchipanov N. A. and Oleinichenko V. Yu. 1992a. On ecology of the white-toothed shrew (Crocidura leucodon). 1. Behaviour, territory use and reproduction of the white-toothed shrew in experiment. A hypothetic scheme of the population density regulation. Zoologicheskii Zhurnal 71: 69-84.

Shchipanov N. A. and Oleinichenko V. Yu. 1992b. On ecology of the white-toothed shrew (Crocidura leucodon). 2. A field study of spatially-ethological structure of the white-toothed shrew population. Two types settlements. Population numbers regulation in dependence on proportion of the settlements with different spatial structures. Zoologicheskiï Zhurnal 71: 110-122.

Leszek RYCHLIK, Mammal Research Institute, Polish Academy of Sciences, 17-230 Białowieża, Poland 